\title{
PUBLICATIONS
}

\section{Published Papers. Vol. I}

\author{
Mathematical Papers Read at the International \\ mathematical Congress Held in Connection \\ WITH THE WORLD's Columbian ExPo- \\ sition, Chicago, 1893.
}

EDITED BY THE COMMITTEE OF THE CONGRESS :
E. Hastings Moore, Oskar Bolza, Heinrich Maschke, HeNRY S. White.

Containing papers by Bolza, Burkhart, Capelli, Cole, Dyck, Echols, Eddy, Fricke, Halsted, Heffter, Hermite, Hilbert, Hurwitz, Klein, Krause, Lemoine, Lerch, Macfarlane, Martin, Maschke, Meyer, Minkowski, Moore, Netto, Noether, D'Ocagne, Paladini, de Perott, Pervouchine, Pincherle, Pringsheim, Sawin, Schlegel, Schoenflies, Stringham, Study, Taber, Weber, Weyr.

Published for the Society by The Macmillan Company, 1896. 8 vo, pp. 411. Price, $\$ 4.00$; to members of the Society, $\$ 3.00$. 